\title{
Blick zurück nach vorn
}

\section{Was wir mit dem ersten Armutsbericht von 1989 angestoßen haben - und was wir immer noch nicht erreicht haben}

Josef Schädle

Josef Schädle ist stellvertretender Vorsitzender des Paritätischen Wohlfahrtsverbandes - Gesamtverband e. V. und einer der Autoren des Armutsberichts "... wessen wir uns schämen müssen in einem reichen Land ... « aus dem Jahre 1989.

Internet http://www.der-paritaetische.de
Der erste bundesweite Armutsbericht des Paritätischen Wohlfahrtsverbandes hat vor zwanzig Jahren das Thema von Armut und Unterversorgung auf die gesellschaftliche Tagesordnung gesetzt und schließlich auch die Politik zum Handeln gezwungen. Ein Initiator und Mitautor des Armutsberichts aus dem Jahre 1989 zieht eine Bilanz dessen, was erreicht wurde und was nicht.

Über zwanzig Jahre ist es nun her, dass ich - wie die anderen Autorinnen und Autoren des ersten Paritätischen Armutsberichts - gespannt vor dem Fernseher saß. Der damalige sozialwissenschaftliche Referent Dr. Ulrich Schneider hatte gleich nach der Pressekonferenz am Vormittag des 9. November 1989 alle angerufen und mitgeteilt: »Alles super gelaufen. Stellt Euch vor, sogar ein Team der $>\mathrm{Ta}$ gesschau< war da! « Am Abend des 9. November 1989 hat es der Paritätische Wohlfahrtsverband tatsächlich das erste Mal ins Fernsehen geschafft. Doch die Meldung des Tages war eine andere, wie wir alle wissen. Und ich war bewegt, überwältigt und sprachlos wie alle, die an diesem Abend vor dem Fernseher Zeugen dieses historischen Moments werden durften.

Aber: Unsere kleine Meldung zum Armutsbericht, zwischen Schabowskis historischer Pressekonferenz und der » Großen Rentenreform ", war dennoch ein Riesenerfolg. Auch wenn das Verbandslogo, wie ich verraten darf, von der » Tagesschau « verkehrt herum eingeblendet wurde, so waren wir doch mächtig stolz an diesem Abend. Ein Tabu war gebrochen. Mit unserem Armutsbericht hatten wir es tatsächlich geschafft, Begriff und Ausmaß der Armut in Deutschland als Thema erstmals erfolgreich in die Öffentlichkeit zu transportieren.

In Wirklichkeit waren wir ja, so muss ich zugeben, gar nicht die Ersten, die einen Armutsbericht vorlegten. Prof. Dr. Richard Hauser erinnerte uns in Vorbe- reitung auf die Fachtagung zum 20-jährigen Jubiläum des ersten Armutsberichts des Paritätischen zu Recht daran, dass er gemeinsam mit anderen im Rahmen des EG-Programms zur Bekämpfung der Armut bereits im Jahr 1981 einen Nationalen Armutsbericht für Deutschland erstellt hatte. Fakt aber ist: Im politischen Raum war »die Armut « Ende der 1980er Jahre noch nicht angekommen.

\section{An der Basis herrschte Sachkenntnis}

Während wir vor Ort die Auswirkungen von Massenarbeitslosigkeit und zunehmender Armut tagtäglich hautnah erlebten, stritt die Politik darüber, ob wir es in der Bundesrepublik wirklich mit einer wachsenden Armut zu tun hätten oder nicht. Während unsere Mitgliedsorganisationen, seien es Arbeitslosen- und Sozialhilfeinitiativen, Behindertenorganisationen oder Vereine für ambulante Pflege, die Not bestimmter Bevölkerungsgruppen Tag für Tag vor Augen hatten, hielt sich die Politik mit der Diskussion auf, wie und ob man Armut denn überhaupt definieren könne. Während wir alles taten, um Arbeitslosen, Behinderten, Alleinerziehenden, Ausländern, Flüchtlingen und anderen betroffenen Menschen durch Beratung, Unterstützung, Förderung und sozialanwaltschaftliche Vertretung zu helfen, tat die Politik - nichts.

Die paradoxen politischen Diskussionen waren für uns nicht nachvollziehbar. Die zu beobachtende Tendenz, Armut zu verdrängen, sie schlicht zu leugnen, machte uns wütend. Die Forderung nach einer institutionalisierten Armutsberichterstattung stand seit Jahren unerfüllt im Raum.

Also haben wir uns des Themas angenommen. Im Jahre 1986 hat der Beirat des Paritätischen Wohlfahrtsverbandes auf mehreren Ebenen entsprechende Aktivitäten angestoßen, deren Ergebnis un- 
ter anderem ein erstes Themenheft der Blätter der Wohlfahrtspflege war (»Armutsberichterstattung in der Bundesrepublik «, Blätter der Wohlfahrtspflege 11/1986). Schließlich wurde die Idee eines gemeinsamen Armutsberichts aller Spitzenverbände in die Bundesarbeitsgemeinschaft der Freien Wohlfahrtspflege hineingetragen und von allen Partnern aufgenommen. Der Vorstand des Paritätischen berief daraufhin eine Arbeitsgruppe, bestehend aus Vertretern des Gesamtverbandes, der Landesverbände und Mitgliedsorganisationen, die im April 1988 ihre Arbeit aufnahm. Im Februar 1989 stellte sich heraus, dass das Vorhaben als gemeinsames Projekt der Freien Wohlfahrtspflege aus verschiedenen Gründen nicht zu realisieren war. Der Paritätische entschloss sich daraufhin, das Vorhaben allein zu verwirklichen.
Wir waren uns dabei bewusst, was wir leisten können und was nicht. In unserem Armutsbericht haben wir die im Verband vorliegenden Erfahrungen mit allgemein zugänglichen statistischen Daten verknüpft. Bislang standen vereinzelte Erfahrungsberichte von Betroffenen und Praktikern neben häufig schwer lesbaren und in Fachzeitschriften versteckten Statistiken. Wir haben die Erkenntnisse, die vorlagen, zu einem ersten, wenn auch unvollständigen Gesamtbild zusammengefügt. Wir sind für verschiedene Bevölkerungsgruppen, von denen wir aus Erfahrung wussten, dass sie in besonderer Weise von sozialer Not bedroht sind, der Frage nachgegangen, was wir über die Lebenslagen dieser Menschen wissen. Die Wahl dieses Lebenslagenansatzes war klug und wurde später durch die Politik aufgegriffen, denn durch den Lebensla-

\section{»Das Thema >Armut< ist kein Tabu mehr, aber Armut haben wir immer noch «}

Und wir waren schnell damals. Ein gutes halbes Jahr später lag der Bericht vor. Diskutiert worden war genug. Mein besonderer Dank gilt den Blättern der Wohlfahrtspflege, die eine schnelle und unkomplizierte Veröffentlichung ermöglichten.

\section{Armutsreport machte Druck}

Wir wussten genau, was wir wollten. Uns war klar: Wir müssen die Armut in Deutschland sichtbar machen. Wir müssen Politik und Öffentlichkeit einen Eindruck ihres Ausmaßes und ihrer Qualität vermitteln, um der Gefahr der Verleugnung und Verzerrung sachlich entgegenzutreten. Wir müssen auf die vielen Leerstellen im Wissen um die Armut aufmerksam machen. Und wir müssen der Forderung nach einer offensiven, rationalen Armutspolitik auf der Grundlage einer institutionalisierten Armutsberichterstattung Nachdruck verleihen. Der Armutsbericht sollte die Bundesregierung endlich zum Handeln bewegen. genansatz wurde Armut und die Bedeutung für den Einzelnen greifbar.

Die damalige Bundesregierung sah jedoch auch nach der Veröffentlichung unseres Berichts keinen Handlungsbedarf und hielt an der Auffassung fest, eine Armutsberichterstattung sei nicht möglich. Es sollte zwölf weitere Jahre dauern, bevor mit dem »1. Armuts- und Reichtumsbericht « der Bundesregierung im Jahr 2001 der Grundstein für eine institutionalisierte Armutsberichterstattung gelegt wurde.

Zwanzig Jahre sind eine lange Zeit. Und Jubiläen sind immer ein guter Anlass, um zurückzublicken, Bilanz zu ziehen und gleichzeitig nach vorne zu schauen. Was haben wir erreicht? Was ist geworden aus den Zielen, die wir uns damals gesteckt hatten, die uns motivierten, den Armutsbericht zu schreiben?

Heute, zwanzig Jahre später, ist Armut kein Tabu mehr. Aber das Problem der Armut, so viel steht fest, haben wir nicht in den Griff bekommen. Infrastrukturell hat sich einiges verbessert für verschiedene Betroffenengruppen. Aber die damals beschriebenen, besonders von Armut bedrohten Gruppen sind dieselben geblieben. Für die Gruppe der psychisch Kranken kann ich es konkret sagen: Auch heute noch ist mehr als die Hälfte der chronisch psychisch Kranken auf fremde materielle Hilfe und Transferleistungen angewiesen. Und die reichen vorne und hinten nicht. Aber auch für die anderen Gruppen hat sich die Situation kaum verbessert: Mehr als jede dritte Alleinerziehende und ihre Kinder sind von Armut bedroht. Menschen mit Migrationshintergrund sowie Familien mit Kindern gehören nach wie vor zu den am stärksten von Armut betroffenen Gruppen.

\section{... wessen wir uns immer noch schämen müssen in einem rechen Land ...}

Die Ungleichheit in unserer Gesellschaft hat eher zu- als abgenommen. Die reichsten zehn Prozent der Deutschen besitzen fast zwei Drittel des gesamten Volksvermögens, die ärmste Hälfte dagegen fast nichts. Am unteren Ende der Vermögensskala sind bereits rund acht Prozent der Haushalte völlig überschuldet.

Die Gesellschaft ist gespalten, die Einführung von Hartz IV hat diesen Spaltungsprozess verschärft und beschleunigt. Und dabei reichen die Folgen der Einkommensarmut weit über das Materielle hinaus, wie wir schon damals deutlich betont haben: Armut ist eine in verschiedener Hinsicht belastende Lebenssituation, die bestimmt wird durch Mangel und Ausgrenzung, durch fehlende Zukunftschancen und Teilhabemöglichkeiten in der gesamten Lebenslage: von den Wohnverhältnissen, über die Bildung bis zur Gesundheitsversorgung.

»...wessen wir uns schämen müssen in einem reichen Land ... « haben wir unseren Armutsbericht damals überschrieben. Wir haben bewusst die provokante Frage in den Raum gestellt: Wo liegt die "Schamgrenze« unserer Gesellschaft? Was wollen wir als reicher Sozialstaat an sozialen Ungleichheiten hinnehmen? Und wir haben gesagt: Was wir an Ungleichheiten nicht hinzunehmen bereit sind, und wessen wir uns schämen müssen in einem reichen Land, dies ist Armut.

Es scheint an der Zeit, die Frage von damals neu zu stellen. 